\title{
ARTICLE OPEN \\ Structural prediction of stabilized atomically thin tin layers
}

\author{
Pedro Borlido $\mathbb{C}^{1}$, Ahmad W. Huran², Miguel A. L. Marques ${ }^{2}$ and Silvana Botti ${ }^{1}$
}

The family of group IV two-dimensional materials shows a rich variety of structural, electronic and topological properties. Only graphene is stable in the honeycomb structure, while buckling and dumbbell configurations stabilize silicene and germanene. Here we investigate from first principles the lowest-energy atomic arrangements of atomically-thin tin layers. Our calculations are performed with a very efficient method for global structural prediction, combined with constrains that enforce the desired onedimensional confinement and include the effect of strain due to the substrate. We discover a series of new structures that span a large range of atomic densities and are considerably more stable than hexagonal single- or double-layer stanene, as well as dumbbell structures. The ground state, a metallic double layer with a square lattice that lies $295 \mathrm{meV} / \mathrm{atom}$ below honeycomb stanene and only $149 \mathrm{meV} /$ atom above bulk $a$-tin, is akin to the atomic arrangement of a layer of romarchite tin oxide. Due to its enhanced stability with respect to honeycomb stanene, we propose that this structure can be easily synthesized on appropriate lattice-matched metallic substrates.

npj 2D Materials and Applications (2019)3:21 ; https://doi.org/10.1038/s41699-019-0103-9

\section{INTRODUCTION}

Since the discovery and characterization of graphene, ${ }^{1,2}$ twodimensional (2D) materials have been the subject of an increasing body of research. In flatland we can find several interesting systems with exotic properties driven by quantum-size effects. Besides graphene, the $2 \mathrm{D}$ archetype, the most studied systems have been 2D polytypes of other group-IV elements (silicene, germanene, and stanene), hexagonal boron nitride, borophene, phosphorene, bismuthene, and transition-metal dichalcogenides. ${ }^{3,4}$

Here we want to focus our attention on 2D tin. In fact, this system has particularly appealing properties. For example, fewlayer stanene has been experimentally determined to be a superconductor with a critical temperature between 0.6 and $2 \mathrm{~K}$ (depending on the number of layers). ${ }^{5}$ The high reactivity of the buckled honeycomb monolayer has been predicted to offer low temperature trapping and dissociation of common air pollutants, such as $\mathrm{NO}_{x}, \mathrm{SO}_{x}$ and $\mathrm{CO}_{x} .{ }^{6}$ Moreover, due to the position of $\mathrm{Sn}$ in the periodic table, spin-orbit effects become relevant in tin crystals, opening the door for topological behavior. Theoretical studies have indeed predicted that buckled stanene is a quantum spin-Hall insulator, ${ }^{7,8}$ with a band gap of $0.1 \mathrm{eV}$ that can be increased to $0.3 \mathrm{eV}$ by chemical functionalization, ${ }^{9}$ allowing to preserve the topological properties up to almost room temperature.

Tin is a rather mundane chemical element belonging to the same group as carbon. However, due to the increasing size of the atomic core as we descend group-IV, the tetrahedral coordination, driven by $s p^{3}$ hybridization, becomes progressively more and more favored than $s p^{2}$-like planar configurations. As such, there is in nature no silicon, germanium, or tin analog of bulk graphite, which hinders the preparation of atomically thin samples for these elements via exfoliation. Fortunately, more advanced preparation techniques have already led to the experimental realization of honeycomb silicene, germanene, and stanene on adequate metallic substrates, e.g., on Ag or Au (111) surfaces. ${ }^{10-13}$

The increase of $s p^{3}$ hybridization has a number of other consequences. For example, $\mathrm{Si}, \mathrm{Ge}$, and $\mathrm{Sn}$ planar honeycomb structures are dynamically unstable, ${ }^{14}$ hence these materials prefer to adopt a buckled hexagonal motif. Furthermore, the relative buckling height increases along the group from $0.44 \AA^{14}$ for $\mathrm{Si}$, to $0.64 \AA^{14}$ for $\mathrm{Ge}$, and finally to $0.85 \AA^{15}$ for $\mathrm{Sn}$. This also seems to justify the impossibility to experimentally build freestanding atomic sheets from group-IV elements other than carbon. ${ }^{16}$

Another direct consequence is that the stability of these elemental monolayers is greatly enhanced by the addition of adatoms or by building multi-layered structures. Indeed, in the case of silicene and germanene, the addition of atoms of the same element on top and under the 2D hexagonal plane, forming a socalled dumbbell, leads to motifs that can be $218 \mathrm{meV} / \mathrm{atom}$ lower in energy than the parent honeycomb structure. ${ }^{17,18}$ To some extent, tin exhibits a similar behavior, as some dumbbell configurations were shown to be at least $180 \mathrm{meV} / \mathrm{atom}$ lower than stanene. ${ }^{15}$ Additionally, a $\mathrm{MoS}_{2}$-like structure was found to be even more stable, 230 meV/atom lower than stanene. ${ }^{15}$

Layer stacking also gives rise to a stabilization effect. This becomes evident if one considers buckled stanene as a reconstruction of an isolated $a$-tin (111) surface layer. In this picture, an $a$-tin-like supercell can be built from the AB stacking of buckled stanene with a layer separation of $2.88 \AA$. The $A B$ stacked bilayer of buckled stanene is already $122 \mathrm{meV} /$ atom below the monolayer. This effect could indeed explain the possibility of fabricating few-layer free-standing stanene. ${ }^{19}$

Interesting questions now arise: what happens when we add atoms to a stanene monolayer? Will they bond to the atoms of the honeycomb lattice in order to form dumbbells, or will a new layer of stanene be formed? Does tin prefer completely new

\footnotetext{
${ }^{1}$ Institut für Festkörpertheorie und -optik, Friedrich-Schiller-Universität Jena, Max-Wien-Platz 1, 07743 Jena, Germany and ${ }^{2}$ Institut für Physik, Martin-Luther-Universität HalleWittenberg, D-06099 Halle, Germany

Correspondence: Silvana Botti (silvana.botti@uni-jena.de)
}

Received: 23 October 2018 Accepted: 7 April 2019

Published online: 13 May 2019 
geometrical arrangements, still unreported for the other group-VI compounds? Of course, the situation becomes even harder to predict if we want to account for the effect of substrate constraints.

\section{RESULTS AND DISCUSSION}

To shed light onto this intriguing problem, we decided to investigate extensively the possible morphological arrangements of tin layers with a thickness of few ångström. Our tool is global structural prediction, ${ }^{20}$ and more specifically the minima-hopping method. ${ }^{21,22}$ This is an efficient and successful technique ${ }^{23,24}$ to perform a completely unbiased exploration of the BornOppenheimer potential-energy surface. Often global structural prediction algorithms input energies and forces from a densityfunctional theory (DFT) calculation. It is true that DFT is probably the best electronic structure method for solids on the market, as it combines an extraordinary precision with a rather modest computational effort. However, our runs require hundreds of thousands of energy and force evaluations, making the use of DFT exceedingly slow when the size of the unit cell increases. As a workaround, we use a two-step procedure. First, we perform global structural prediction runs with energies and forces calculated with density-functional tight-binding, as implemented in the DFTB + package. ${ }^{25}$ We then select the most relevant structures and re-optimize them with DFT. ${ }^{26,27}$

Our results are summarized in Fig. 1, where we plot the DFT energy per atom as a function of the area for the most interesting structures stemming from the minima-hopping runs. More detailed structural data, together with band-structures, can be found as Supplementary Information. Each curve was calculated by applying an equibiaxial strain to the corresponding optimized structure. Colored curves represent the frontier of the phase diagram, and are labeled from Sn-I to Sn-IX in increasing order of the position of the minimum. The zero of energy is chosen to be $\mathrm{Sn}-\mathrm{I}$ in mechanical equilibrium. For comparison, single-layer stanene appears very unstable, with an energy of $295 \mathrm{meV} / \mathrm{atom}$ above the ground state $\mathrm{Sn}-\mathrm{I}$ and with an area per atom of $9.5 \AA^{2}$ / atom, buckled $A B$ bilayer stanene is at $174 \mathrm{meV} /$ atom with an area per atom of $4.5 \AA^{2}$ /atom, buckled ABA trilayer stanene lies at $98 \mathrm{meV} /$ atom in this energy scale with an area per atom of $3.18 \AA^{2}$ /atom (outside the range displayed in Fig. 1). Note that bulk $a-\mathrm{Sn}$ is only $149 \mathrm{meV} /$ atom more stable than $\mathrm{Sn}-\mathrm{l}$.

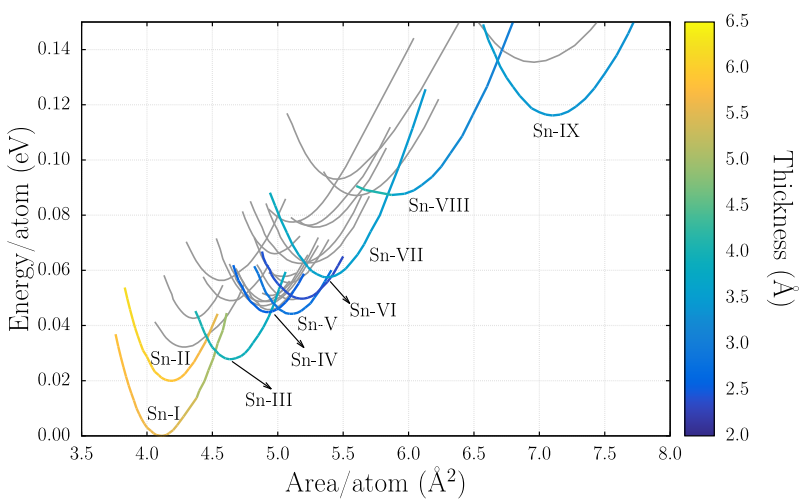

Fig. 1 Energy per atom as a function of the area per atom for twoatom thick tin structures. All values are plotted with respect to the energy minimum of Sn-I. Gray curves represent a selection of structures obtained from the minima-hopping method that do not form a boundary for the energy curve. Thickness is measured as the vertical distance between the top- and bottom-most atoms of each structure. For reference, the buckled honeycomb mono-, $A B$ bi- and ABA trilayers of stanene are located 296,174 and $98 \mathrm{meV} / \mathrm{atom}$ above Sn-l, respectively, while bulk $a$-tin is merely $149 \mathrm{meV} / \mathrm{atom}$ below it
From Fig. 1 we can immediately see how buckling stabilizes layered tin phases. In fact, the lowest energy structures are also the thicker ones. To obtain thinner layers requires larger areas/ atoms, that can be achieved, e.g., through strain.

The most stable structure we found, i.e., the ground-state of a quasi-two-dimensional layer of tin, lies on the left of Fig. 1 with an area of $4.07 \AA^{2}$ /atom. This structure, that can be described by a square lattice with 8 atoms in the primitive unit cell, is a staggering $174 \mathrm{meV} /$ atom below buckled bilayer stanene. Surprisingly, Sn-I does not exhibit the familiar hexagonal honeycomb arrangement of graphene, silicene, germanene, or stanene. Instead, it is a bilayer of corrugated squares (see Fig. 2), with an atomic arrangement akin to the one of $\mathrm{Sn}$ and $\mathrm{O}$ in a layer of the (layered) romarchite tin oxide. Each layer features tin atoms in a stretched tetrahedral coordination occupying alternating sites, while the other sites are occupied with atoms in a pyramidal configuration with a square base. The enhanced stability of Sn-I with respect to buckled stanene can be understood in light of the more favorable tetrahedral coordination of tin atoms. These tetrahedra are of lower symmetry than those that build up $a$-tin, as their faces are isosceles triangles rather than equilateral ones. We remark that Sn-I is more stable than both bilayer and trilayer buckled stanene, appearing therefore as an island of stability for atomically-thin films made of tin, in an area/atom range intermediate between the one of bilayer and trilayer structures.

As it turns out, Sn-I (like all the other phases discussed below) is an excellent conductor with a strong metallic character (see Fig. 3 and Supplementary Information), due to several bands crossing the Fermi energy. Not surprisingly, as there is no honeycomb lattice, no Dirac cone can be found at the Fermi level.

In ref. ${ }^{12}$ the growth of an hexagonal bilayer of tin on $\mathrm{a} \mathrm{Bi}_{2} \mathrm{Te}_{3}$ (111) surface is reported. We remember that buckled hexagonal bilayers of tin have an energy per atom $174 \mathrm{meV} /$ atom higher than Sn-l, and more than $300 \mathrm{meV} / \mathrm{atom}$ higher than bulk $a$-Sn. Also silicene lies more than $400 \mathrm{meV} / \mathrm{atom}$ above the diamond phase, but it has been experimentally synthesized thanks to its stabilization on substrates. Supporting metallic substrates can indeed stabilize silicene by donating electrons, while preserving a moderately small interaction between the monolayer and surface atoms. We expect therefore that also $\mathrm{Sn}$-I should be synthesizable on appropriate metallic substrates. In view of the in-plane square lattice displayed by Sn-I, we can suggest some possible metallic substrates with square surface lattices and a small lattice mismatch. Surfaces of $\mathrm{Ag}$ and $\mathrm{Au}$ remain good candidates if we replace the (111) surface with the (100) surface. Ag (100) and Au (100) squared lattices have a lattice mismatch of 2.7 and $2.9 \%$, respectively, corresponding to an equivalent area/atom of 4.33 and $4.35 \AA^{2}$ /atom, very close to the stability minimum, and well inside the stability interval of Sn-I. Even closer to the equilibrium area/atom are Al (100) and Pt (100) surfaces, with lattice mismatches of 0.3 and $1.8 \%$ only.

To ascertain the dynamical stability of free-standing Sn-I we calculated the phonon band-structure using the finite differences method as implemented in the PHONOPY package. ${ }^{28}$ We used the Perdew-Zunger ${ }^{29}$ local-density approximation, as this functional was shown to outperform PBE for phonon-related properties. ${ }^{30}$ Unlike the monolayer of romarchite tin oxide, which is dynamically unstable, ${ }^{31} \mathrm{Sn}-\mathrm{I}$ has only real phonon frequencies (see Fig. 3). As expected considering the large mass of Sn, phonon frequencies are rather low, and consistently within the same energy range of the phonon frequencies of $a$ - or $\beta-\mathrm{Sn}^{32}$

The structural motif observed in Sn-I seems to be favored for structures with up to $4.5 \AA^{2} /$ atom. Sn-Il, $19 \mathrm{meV} /$ atom almost directly above $\mathrm{Sn}-\mathrm{l}$, displays a corrugation pattern modified along one of the in-plane directions, becoming asymmetric in a sort of sawtooth-like structure. Structural similarities to $\mathrm{Sn}$-I are more apparent in Sn-III (27 meV/atom above Sn-I). This structure can be seen as a reconstruction of the $\mathrm{Sn}-\mathrm{I}$ monolayer, exhibiting sharper 


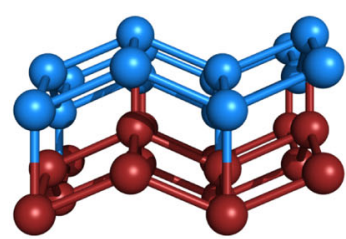

(a) Sn-I

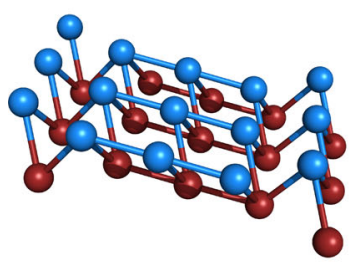

(e) Sn-V

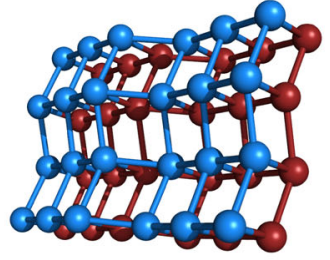

(b) Sn-II

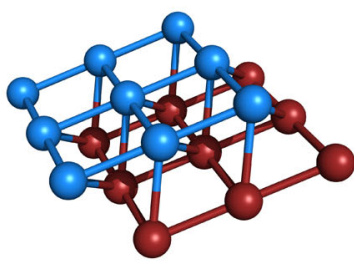

(f) Sn-VI

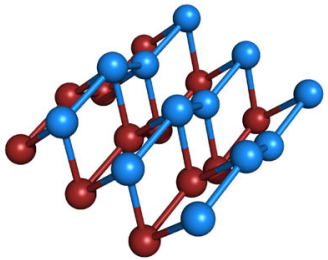

(c) Sn-III

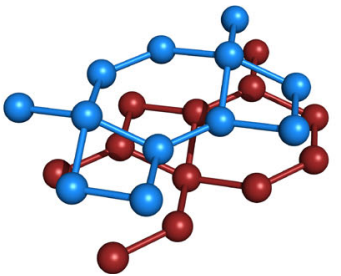

(g) Sn-VII

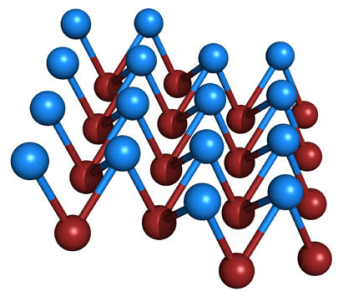

(d) Sn-IV

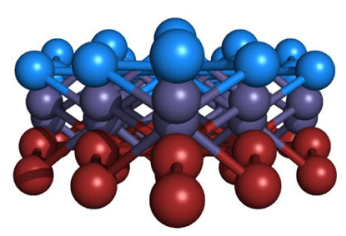

(h) Sn-VIII

Fig. 2 Crystal structure of the polymorphs mentioned in the main text. Sn-IX is not shown since it has been extensively investigated in the literature. Atoms are colored using a scale from blue to red according to their relative position at the top or bottom of the structure, respectively

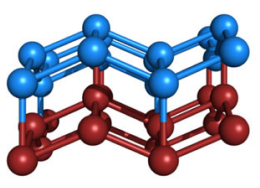

(a)

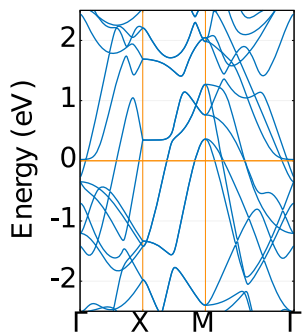

(c)

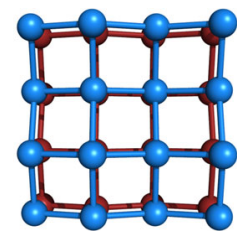

(b)

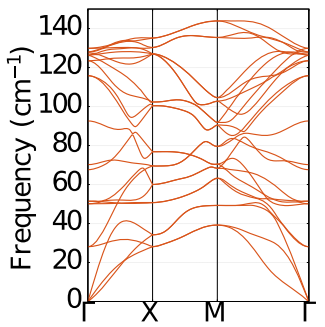

(d)
Fig. 3 a Side and $\mathbf{b}$ top views of Sn-I, together with the calculated $\mathrm{PBE}$ electronic band structure $\mathbf{c}$, and phonon bands $\mathbf{d}$

corrugation patterns but a smaller thickness of approximately $4.0 \AA$ (compared to the $5.6 \AA$ of $S n-I)$.

At lower values of atomic density, new types of structure becomes favored. The Sn-IV phase, $44 \mathrm{meV} /$ atom above Sn-I, is composed of buckled hexagons. The buckling height is $2.57 \AA$, almost three times larger than the one of stanene. In this picture, $\mathrm{Sn}$ IV can be seen as the result of a simple transformation of buckled stanene, namely, an increment in the buckling height accompanied with shrinkage of the lattice constant, forming what is usually known as the high-buckled (HB) honeycomb structure. Along the aforementioned transformation path, we estimated the internal energy barrier to be $227 \mathrm{meV} / \mathrm{atom}$, with the transition structure exhibiting a buckling height of $1.66 \AA$. Unlike the dynamically unstable $\mathrm{HB}$ silicene and germanene. ${ }^{14} \mathrm{HB}$ stanene is a true local minimum of the potential energy surface (see Supplementary Information). We note that the increased buckling is accompanied by an enhanced electron localization on the atomic sites.

Between 5.0 and $5.5 \AA^{2}$ /atom we find three structures: Sn-V, Sn-VI, and $\mathrm{Sn}$-VII. The Sn-V phase, $43 \mathrm{meV} /$ atom above $\mathrm{Sn}-\mathrm{I}$, consists of alternating corrugated sections that meet at distorted buckled hexagons, similar to the ones encountered in Sn-IV. At slightly higher formation energies we find $\mathrm{Sn}-\mathrm{Vl}$, formed by two shifted planar networks of rhombi. The following minimum, Sn-VIl, is a 6-4 haeckelite double-layer, located $57 \mathrm{meV} /$ atom above Sn-I. An interesting feature of this structure is that its monolayer analog is extremely unstable, as it lies $430 \mathrm{meV} / \mathrm{atom}$ above Sn-l. Therefore, the formation of the bilayer results in a $373 \mathrm{meV} / \mathrm{atom}$ stabilization.

Finally, we refer to the Sn-VIII and Sn-IX allotropes, which have geometrical elements in common. Sn-IX is found at around $7.0 \AA^{2}$ / atom, $115 \mathrm{meV} /$ atom above $\mathrm{Sn}$-l. This structure is well-known for 2D materials of group IV, and it is often called the large honeycomb dumbbell. ${ }^{17}$ Curiously, it is a low-energy structure for both silicon and germanium. Furthermore, previous studies on Sn predict it to be dynamically stable and also a quantum spin Hall insulator. ${ }^{33}$ Sn-VIII can be derived from the Sn-IX structure, as it consists of a series of large honeycomb dumbbell ribbons (with the dumbbells along the edges), tilted collectively along their translational axis, and joined at the facing dumbbells (see Supplementary Information). The additional coordination deriving from dumbbell atoms is enough to lower this structure by $30 \mathrm{meV} /$ atom with respect to $\mathrm{Sn-IX}$.

We would also like to comment on one last structure of 2D-tin that has been recently proposed in the literature, specifically the $\mathrm{MoS}_{2}$ stanene variant. ${ }^{15}$ This structure lies at $3.12 \AA^{2} /$ atom, circa $50 \mathrm{meV} /$ atom below the large honeycomb dumbbell, ${ }^{15}$ but still $60 \mathrm{meV} /$ atom above Sn-I (an energy range with a large density of possible structures).

In conclusion, we performed extensive global structural prediction calculations for quasi-two-dimensional tin, in a large range of atomic densities, focusing on a layer thickness comparable to the one of buckled bilayer stanene. More than 100000 minima were found, from which we selected the most interesting for further analysis. Our results show a complex landscape, with several different geometrical arrangements competing in the low-energy regime. The most stable polymorph of 2D bilayer tin, that we named $\mathrm{Sn}-\mathrm{I}$, has a square lattice and metallic character, with several electronic bands crossing the Fermi level. Its structure is reminiscent of a layer of romarchite tin oxide, where both tin and oxygen sites are occupied in this case by tin. This arrangement allows for a tetrahedral coordination of the Sn atoms, therefore favoring a large $s p^{3}$ hybridization. Thanks to the much smaller energy difference to bulk $a$-tin (Sn-I is only $149 \mathrm{meV}$ / atom higher) we believe that this structure could be produced 
experimentally, using e.g., the stabilizing effect of almost latticematched Ag (100), Au (100), Pt (100), or Al (100) substrates.

\section{METHODS}

\section{Crystal-structure prediction calculations}

Crystal-structure prediction calculations were performed using a constrained version of the minima-hopping method. ${ }^{21,22}$ We have already successfully applied this constrained approach to investigate 2D polymorphs of carbon, ${ }^{34}$ silicon, ${ }^{18}$ and germanium. Due to the amount of structures considered, forces and energies were first computed with density-functional tight-binding, as implemented in the DFTB+ package. ${ }^{25}$ For this step, we used home-made high-quality Slater-Koster parameters, specifically fitted to obtain energies and forces with quasi-DFT precision. ${ }^{35}$ The number of calculations also forced us to neglect spin-orbit coupling at this stage, although we verified a posteriori that this is acceptable (see below).

A systematic structural prediction of supported 2D tin, including explicitly its interaction with every possible substrate, is beyond our computational capabilities. We decided therefore to simulate the strain due to the substrate by constraining the unit-cell area of the free-standing tin layers. We performed structural prediction at fixed area, spanning values of the area/atom in a large interval that is compatible with the formation of both monolayer and bilayers of tin. This translates to inverse atomic densities ranging between 3.5 and $8 \AA^{2} /$ atom. We complemented then these fixedarea calculations with unconstrained ones. We considered unit cells containing 2 to $20 \mathrm{Sn}$ atoms. All in all, we obtained more than 100000 local minima, with layer thickness varying between $2.4-5.9 \AA$. At the end we searched for lattice-matched metallic substrates for the identified lowestenergy structures. In this way we assume that the stabilizing effect of the substrate is limited to the donation of electrons to the ultra-thin tin layer, while we neglect the formation of chemical bonds between stanene and the substrate. This choice is essential to make possible an exhaustive largescale exploration of the most stable 2D crystal structures.

\section{DFT calculations}

The most relevant structures were re-optimized with DFT within the projector-augmented wave formalism, as implemented in VASP. ${ }^{26,27}$ The Perdew-Burke-Ernzerhof $(\mathrm{PBE})^{36}$ approximation to the exchangecorrelation functional was used for geometry optimizations and bandstructure calculations. In the direction perpendicular to the layer, we built supercells containing at least $15 \AA$ of vacuum to isolate periodic copies, while in the plane we used a $k$-point set with a density corresponding to a $12 \times 12$ grid for stanene.

All these calculations were performed without taking into account spinorbit coupling, mostly to reduce the computational cost. However, we know that tin is a rather heavy element, with a sizable spin-orbit interaction, that is important in certain contexts. As such, we repeated $a$ posteriori DFT calculations for a series of structures including this term. We found that spin-orbit coupling does have a measurable effect in the formation energy. However, this energy shift is nearly constant for all $2 \mathrm{D}$ structures (55-59 meV/atom), thereby our assumptions are justified.

\section{DATA AVAILABILITY}

All data that support the findings of this study are available in the published article (and in its Supplementary Information files). The Crystal Information Files are also included in the Supplementary Information. Source data for figures are available from the corresponding author upon reasonable request. Supplementary material is available on the journal website.

\section{ACKNOWLEDGEMENTS}

M.A.L.M. and S.B. acknowledge partial support from the DFG though the projects TRR 227 and BO 4280/8-1, respectively. Computational resources were provided by the Leibniz Supercomputing Centre through the projects p1841a and pr62ja.

\section{AUTHOR CONTRIBUTIONS}

M.A.L.M. and S.B. designed and directed the project. A.H. computed Slater-Koster parameters. P.B. performed minima hopping and electronic structure calculations. All authors contributed to the analysis of the data and to the writing of the manuscript.

\section{ADDITIONAL INFORMATION}

Supplementary Information accompanies the paper on the npj 2D Materials and Applications website (https://doi.org/10.1038/s41699-019-0103-9).

Competing interests: The authors declare no competing interests.

Publisher's note: Springer Nature remains neutral with regard to jurisdictional claims in published maps and institutional affiliations.

\section{REFERENCES}

1. Novoselov, K. S. et al. Electric field effect in atomically thin carbon films. Science 306, 666-669 (2004).

2. Novoselov, K. S. et al. Two-dimensional gas of massless dirac fermions in graphene. Nature 438, 197 (2005).

3. Choi, W. et al. Recent development of two-dimensional transition metal dichalcogenides and their applications. Mater. Today 20, 116-130 (2017).

4. Wang, G. et al. Colloquium: Excitons in atomically thin transition metal dichalcogenides. Rev. Mod. Phys. 90, 021001 (2018).

5. Liao, M. et al. Superconductivity in few-layer stanene. Nat. Phys. 14, 344-348 (2018).

6. Takahashi, L. \& Takahashi, K. Low temperature pollutant trapping and dissociation over two-dimensional tin. Phys. Chem. Chem. Phys. 17, 21394-21396 (2015).

7. Liu, C.-C., Jiang, H. \& Yao, Y. Low-energy effective hamiltonian involving spin-orbit coupling in silicene and two-dimensional germanium and tin. Phys. Rev. B 84, 195430 (2011).

8. Fu, B., Abid, M. \& Liu, C.-C. Systematic study on stanene bulk states and the edge states of its zigzag nanoribbon. New J. Phys. 19, 103040 (2017).

9. $\mathrm{Xu}, \mathrm{Y}$. et al. Large-gap quantum spin hall insulators in tin films. Phys. Rev. Lett. 111, 136804 (2013).

10. Vogt, P. et al. Silicene: Compelling experimental evidence for graphenelike twodimensional silicon. Phys. Rev. Lett. 108, 155501 (2012).

11. Dávila, M. E., Xian, L., Cahangirov, S., Rubio, A. \& Lay, G. L. Germanene: a novel two-dimensional germanium allotrope akin to graphene and silicene. New J. Phys. 16, 095002 (2014).

12. Zhu, F.-f. et al. Epitaxial growth of two-dimensional stanene. Nat. Mater. 14, 1020-1025 (2015).

13. Yuhara, J. et al. Large area planar stanene epitaxially grown on $\mathrm{Ag}$ (111). $2 D$ Mater. 5, 025002 (2018).

14. Cahangirov, S., Topsakal, M., Aktürk, E., Şahin, H. \& Ciraci, S. Two- and onedimensional honeycomb structures of silicon and germanium. Phys. Rev. Lett. 102, 236804 (2009).

15. Matusalem, F., Marques, M., Teles, L. K. \& Bechstedt, F. Stability and electronic structure of two-dimensional allotropes of group-iv materials. Phys. Rev. B 92, 045436 (2015).

16. Zhang, Y., Rubio, A. \& Le Lay, G. Emergent elemental two-dimensional materials beyond graphene. J. Phys. D: Appl. Phys. 50, 053004 (2017).

17. Cahangirov, S. et al. Atomic structure of the $\sqrt{3} \times \sqrt{3}$ phase of silicene on $\mathrm{Ag}$ (111). Phys. Rev. B 90, 035448 (2014).

18. Borlido, P., Rödl, C., Marques, M. A. \& Botti, S. The ground state of twodimensional silicon. 2D Mater. 5, 035010 (2018).

19. Saxena, S., Chaudhary, R. P. \& Shukla, S. Stanene: atomically thick free-standing layer of 2d hexagonal tin. Sci. Rep. 6, 31073 (2016).

20. Oganov, A. R., Saleh, G. \& Kvashnin, A. G. (eds) Computational Materials Discovery (The Royal Society of Chemistry, UK 2019).

21. Goedecker, S. Minima hopping: An efficient search method for the global minimum of the potential energy surface of complex molecular systems. J. Chem. Phys. 120, 9911-9917 (2004).

22. Amsler, M. \& Goedecker, S. Crystal structure prediction using the minima hopping method. J. Chem. Phys. 133, 224104 (2010).

23. Sarmiento-Prez, R., Cerqueira, T. F. T., Körbel, S., Botti, S. \& Marques, M. A. L. Prediction of stable nitride perovskites. Chem. Mater. 27, 5957-5963 (2015).

24. Cerqueira, T. F. T. et al. Identification of novel $\mathrm{cu}$, ag, and au ternary oxides from global structural prediction. Chem. Mater. 27, 4562-4573 (2015).

25. Aradi, B., Hourahine, B. \& Frauenheim, T. DFTB+, a sparse matrix-based implementation of the DFTB method. J. Phys. Chem. A 111, 5678-5684 (2007).

26. Kresse, G. \& Furthmüller, J. Efficient iterative schemes for ab initio totalenergy calculations using a plane-wave basis set. Phys. Rev. B 54, 11169-11186 (1996).

27. Kresse, G. \& Joubert, D. From ultrasoft pseudopotentials to the projector augmented-wave method. Phys. Rev. B 59, 1758-1775 (1999).

28. Togo, A. \& Tanaka, I. First principles phonon calculations in materials science. Scr. Mater. 108, 1-5 (2015). 
29. Perdew, J. P. \& Wang, Y. Accurate and simple analytic representation of the electron-gas correlation energy. Phys. Rev. B 45, 13244-13249 (1992).

30. He, L. et al. Accuracy of generalized gradient approximation functionals for density-functional perturbation theory calculations. Phys. Rev. B 89, 064305 (2014).

31. Mounet, N. et al. Two-dimensional materials from high-throughput computational exfoliation of experimentally known compounds. Nat. Nanotechnol. 13, 246 (2018).

32. Na, S.-H. \& Park, C.-H. First-principles study of structural phase transition of Sn. J. Korean Phys. Soc. 56, 494-497 (2010).

33. Tang, P. et al. Stable two-dimensional dumbbell stanene: A quantum spin hall insulator. Phys. Rev. B 90, 121408 (2014).

34. Borlido, P., Steigemann, C., Lathiotakis, N. N., Marques, M. A. \& Botti, S. Structural prediction of two-dimensional materials under strain. 2D Mater. 4, 045009 (2017).

35. Huran, A. W., Steigemann, C., Frauenheim, T., Aradi, B. \& Marques, M. A. L. Efficient automatized density-functional tight-binding parametrizations: Application to group iv elements. J. Chem. Theory Comput. 14, 2947-2954 (2018).
36. Perdew, J. P., Burke, K. \& Ernzerhof, M. Generalized gradient approximation made simple. Phys. Rev. Lett. 77, 3865-3868 (1996)

(i) Open Access This article is licensed under a Creative Commons Attribution 4.0 International License, which permits use, sharing adaptation, distribution and reproduction in any medium or format, as long as you give appropriate credit to the original author(s) and the source, provide a link to the Creative Commons license, and indicate if changes were made. The images or other third party material in this article are included in the article's Creative Commons license, unless indicated otherwise in a credit line to the material. If material is not included in the article's Creative Commons license and your intended use is not permitted by statutory regulation or exceeds the permitted use, you will need to obtain permission directly from the copyright holder. To view a copy of this license, visit http://creativecommons. org/licenses/by/4.0/.

(c) The Author(s) 2019 